\title{
Structural and mechanical features of the order-disorder transition in experimental hard-sphere packings
}

\author{
M. Hanifpour, ${ }^{1,3}$ N. Francois, ${ }^{2, *}$ V. Robins, ${ }^{2}$ A. Kingston, ${ }^{2}$ S. M. Vaez Allaei,,${ }^{1,3, \dagger}$ and M. Saadatfar ${ }^{2, \ddagger}$ \\ ${ }^{1}$ Department of Physics, University of Tehran, Tehran 14395-547, Iran \\ ${ }^{2}$ Department of Applied Mathematics, Research School of Physics and Engineering, Australian National University, Canberra, Australia \\ ${ }^{3}$ School of Physics, Institute for Research in Fundamental Sciences (IPM), Tehran 19395-5531, Iran
}

(Received 28 February 2015; published 5 June 2015)

\begin{abstract}
Here we present an experimental and numerical investigation on the grain-scale geometrical and mechanical properties of partially crystallized structures made of macroscopic frictional grains. Crystallization is inevitable in arrangements of monosized hard spheres with packing densities exceeding Bernal's limiting density $\phi_{\text {Bernal }} \approx$ 0.64 . We study packings of monosized hard spheres whose density spans over a wide range $(0.59<\phi<$ 0.72). These experiments harness x-ray computed tomography, three-dimensional image analysis, and numerical simulations to access precisely the geometry and the 3D structure of internal forces within the sphere packings. We show that clear geometrical transitions coincide with modifications of the mechanical backbone of the packing both at the grain and global scale. Notably, two transitions are identified at $\phi_{\text {Bernal }} \approx 0.64$ and $\phi_{c} \approx 0.68$. These results provide insights on how geometrical and mechanical features at the grain scale conspire to yield partially crystallized structures that are mechanically stable.
\end{abstract}

DOI: 10.1103/PhysRevE.91.062202

PACS number(s): 45.70.Cc, 45.70.Qj

\section{INTRODUCTION}

The hard-sphere model provides a powerful paradigm of condensed matter that has led to considerable advances in our understanding of crystallization and vitrification in thermal systems. Fundamentally, it reflects that a simple ingredient, e.g., sphere impenetrability, can produce a remarkably rich phase diagram via entropic interactions only. This fact underlies the continued interest in dense packings problems [1-4]. At the other end of the hard-sphere spectrum, athermal layers or packings made of macroscopic grains have shown striking molecularlike behaviors when they are subjected to vibrational protocols [5-12]. In particular, packings have a propensity to form crystalline structures under strong vibrations. No fundamental understanding has emerged yet for the crystallization in these dissipative materials.

The modern history of experimental studies on monosized hard spheres dates back to the 1950s when Bernal performed a seemingly simple experiment by pouring 8000 glass balls in a box [4]. Bernal reported that the density of disordered packings seems to be bounded by $\phi_{\text {Bernal }} \simeq 0.64$ and that the balls had an average number of six contacts. Numerous experimental and numerical studies have extended Bernal's seminal findings and reported that a stable configuration of frictional monodisperse spheres can exist at densities ranging from $\phi \approx 0.55$ to $\phi \approx 0.64$ [4,13-15]. Some recent numerical studies suggest that this range is even wider [3,16-19]. The possible analogy between the "jamming" (the structural arrest observed in dense amorphous packings with densities $0.55<\phi<\phi_{\text {Bernal }}$ ) and the glass transition has monopolized most of the studies on sphere packings during the past two decades [5,20,21]. In comparison, considerably less is known about the crystallization mechanism in frictional packings

\footnotetext{
*nicolas.francois@anu.edu.au

†smvaez@ut.ac.ir

${ }^{\ddagger}$ mos110@physics.anu.edu.au
}

of spheres. To date, ordered phases observed in granular materials have demonstrated both striking resemblances and profound differences to those observed in thermal systems [7-12]. Accordingly, several questions remain outstanding such as those about the existence of a basic principle governing the order-disorder transition in frictional packings, or the description of the crystal growth at the grain scale.

Here we experimentally study three-dimensional (3D) packings made of cohesionless macroscopic spheres. This material is athermal, dissipative, and naturally forms amorphous structures, yet it can massively crystallize if strongly vibrated or sheared [11,22]. The presence of friction poses a considerable challenge to our understanding of this orderdisorder transition and of packing mechanical stability in general $[5,23-25]$. In the disordered domain $\left(\phi<\phi_{\text {Bernal }}\right)$, polytetrahedral structures, i.e., large aggregates made of weakly distorted tetrahedral patterns of beads, are essential components of amorphous packings [4]. These aggregates can form a rich range of mechanically stable motifs, which are built uniquely on face-adjacent tetrahedra, but none can periodically tile the space $[1,26,27]$. Beyond Bernal's density, $\phi_{\text {Bernal }}$, crystalline clusters inevitably appear in highly monodisperse packings. This emerging order enforces the disappearance of the polytetrahedral aggregates, which are geometrically frustrated. Three successive structural transitions associated with the formation and the evolution of the polytetrahedral aggregates have recently been identified at $\phi_{\text {Bernal }} \approx 0.64$, $\phi_{c} \approx 0.68$, and $\phi \approx 0.72$ [22]. In addition to these topological and geometrical changes, the mechanics of these systems also undergoes remarkable changes when $\phi>\phi_{\text {Bernal }}$ showing a behavior closely entangled with the structural features of the packing [30]. Understanding these relations requires a detailed and accurate investigation of each grain's neighborhood in terms of both their mechanical and geometrical properties $[5,28]$. In particular, recent numerical studies of the crystallization process in idealistic frictionless $3 \mathrm{D}$ packings suggest that the order-disorder transition in packings show some analogies with a first-order phase transition over the 
density range $\left[\phi_{\text {Bernal }}, \phi_{c}\right]$ [29]. Some crucial features of this study have recently been observed in $3 \mathrm{D}$ realistic packings (i.e., weakly polydisperse and frictional) by the authors of the present paper [30]. Here we intend to extend this first experimental study and draw a more exhaustive picture of the structure of partially crystallized packings.

In this contribution, we take advantage of a helical x-ray tomography setup to image 3D packings containing up to 200000 frictional spheres in both cylindrical and spherical containers. We employ a simple vibrational protocol to produce partially crystallized packings. Using state-of-the-art experimental and numerical techniques, we can study the evolution of geometrical and mechanical features at the global and at the grain scale during the disordered-ordered transition.

This paper is organized as follows. Section II describes the experimental setup and the procedure used to obtain partially crystallized packings. Section III details the numerical calculations performed on the experimental data in order to describe the force network with numerical precision and access the tangential forces. Section IV characterizes the geometrical transition when the packing density crosses $\phi_{\text {Bernal }}=0.64$. Section $\mathrm{V}$ focuses on the mechanical features of the crystallization process in terms of grain contacts and the evolution of a topological descriptor.

\section{EXPERIMENTAL DETAILS}

We use monosized acrylic beads (diameter $d=1.00$ and $1.62 \mathrm{~mm}$; polydispersity $=0.025 \mathrm{~mm}$ ), which are packed into large containers (inner diameter $=66 \mathrm{~mm}$ ). The beads are covered with graphite to reduce electrostatic repulsion between them. Nine packings of beads are prepared in cylindrical and spherical containers (see details in Table I). Five of them are produced by simply pouring the beads into the container following the methods described in [14]. The other four, with a density $\phi \gg \phi_{\text {Bernal }}$, are generated according to the vibrational protocol described below.

Our experimental method is based on compaction by an intense fluidization of the packing [22,31]. A batch of beads is initially poured into a container forming a random packing. The container is then placed on a shaker allowing for both vertical and horizontal vibrations. The vibrations are sinusoidal with a frequency set to $f=50 \mathrm{~Hz}$; the vertical component of the acceleration $\gamma_{v}$ is set to be five times

TABLE I. Summary of the experimental packings used in this study. $N$ is the number of grains used for the analysis, $\phi$ is the global packing density, and $D$ is the grain diameter.

\begin{tabular}{lccc}
\hline \hline Container & $N$ & $\phi$ & $D(\mathrm{~mm})$ \\
\hline 1 Cylinder & 26498 & 0.59 & 1.62 \\
2 Cylinder & 25797 & 0.61 & 1.62 \\
3 Cylinder & 27555 & 0.60 & 1.62 \\
4 Cylinder & 27665 & 0.61 & 1.62 \\
5 Cylinder & 156315 & 0.63 & 1.00 \\
6 Cylinder & 31005 & 0.66 & 1.62 \\
7 Cylinder & 216722 & 0.685 & 1.00 \\
8 Spherical & 60205 & 0.685 & 1.00 \\
9 Spherical & 64042 & 0.72 & 1.00 \\
\hline \hline
\end{tabular}

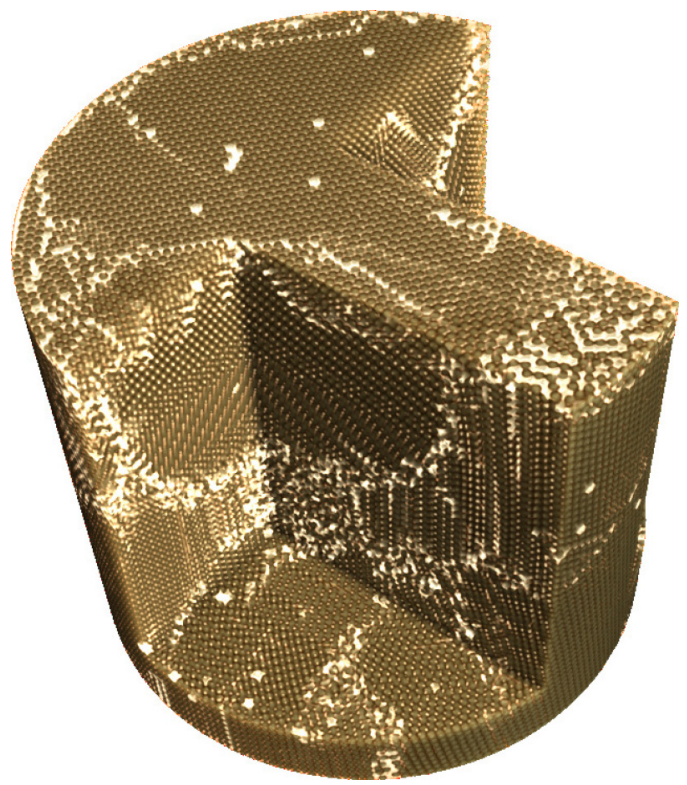

FIG. 1. (Color online) 3D visualization of a partially crystallized packing containing $\approx 200000$ beads. Bright regions indicate the location of disordered aggregates of beads, which have been identified using the $q_{6}$ metrics (see Sec. IV).

larger than the horizontal one $\gamma_{h}$. In these experiments, $\gamma_{v}$ is constant and set to $2.5 \mathrm{~g}$ (where $g$ is the gravitational acceleration). The container is vibrated intensely for $20 \mathrm{~s}$. The resulting packings show substantial crystallization, with a global packing density well beyond Bernal's limit, ranging from 0.66 to 0.72 . The compaction protocol presented here is robust and allows us to consistently generate partially crystallized packings irrespective of the packing container.

Figure 1(a) shows a 3D rendering of a partially crystallized structure. The bright regions correspond to locally disordered aggregates of beads; a disordered core and the boundaries between different crystal domains are signified by the bright regions. Both random and crystalline phases coexist in the packing, yielding nonuniform packing densities across the sample. Helical x-ray computed tomography (XCT) is utilized to digitally access the internal $3 \mathrm{D}$ structure of the packings with a spatial resolution of $\approx 30$ microns [14,22,32-34]. All our analyses have been carried out over the inner region of the packings, four sphere diameters away from the container walls to avoid the boundary effects. These inner regions are then decomposed into nonoverlapping cubical subsets each containing 4000 beads. A total of 94 subsets are produced from all the packings listed in Table I. As a consequence of structural heterogeneity in our partially crystallized packings (see Fig. 1), the 4000-bead subsets cover a wide range of packing densities spanning from $\phi=0.58$ to $\phi=0.73$.

\section{DEM SIMULATION}

The digital representation of each packing is realized using $\mathrm{XCT}$ and a range of postprocessing image analysis techniques. Each grain in the $3 \mathrm{D}$ digital representation (tomogram) is made of a cluster of $\approx 19000$ voxels and each tricubic voxel represents $30^{3} \mu \mathrm{m}$ of space known as the image resolution. As 
a result of this large grain digital representation, the precision with which grain centers are determined is extremely high $\left(<10^{-3} \mu \mathrm{m}\right)$. Similarly, a grain's diameter can be estimated with great precision $\left(\approx 5 \times 10^{-2} \mu \mathrm{m}\right)$. It allows a highly accurate experimental measure of both the grain's center-center distance and the grain overlap. From these high precision experimental data we are able to calculate normal contact forces as low as $\approx 10^{-4} N$, while the average normal force is typically $\left\langle F_{n}\right\rangle \simeq 10^{-1} N$ in our experiments. The tangential component of the contact forces, however, cannot be trivially measured from the experimental data [35].

Force bearing contacts are an extremely sensitive feature of hard-sphere packings [5,28]. Its digital representation will inevitably be affected by intrinsic experimental limitations (finite spatial resolution, binarization segmentation of the tomogram). To further assess the robustness of our mechanical characterization, experimental packing structures are subsequently postprocessed by a discrete element method (DEM) code [36]. We use LIGGGHTS, an open-source DEM code widely used in the granular community [37]. We note that the DEM computation gives access to both components of the intergranular forces. The DEM uses a Hertz-Mindlin model to compute the forces between the grains by virtually relaxing the experimental packings under gravity over a period of a few physical seconds, which takes $\approx 12 \mathrm{~h}$ of simulation time on a 64-processor cluster. The combining of the experimental data from XCT with the DEM simulations allows us to map the network of force bearing contacts with numerical precision. Crucially, the results can be compared with the direct experimental characterization. Additionally, the use of DEM allows us to investigate the sensitivity of our results to the friction coefficient by virtually tuning it (see Sec. V).

The Hertz-Mindlin model characterizes the interaction between frictional grains as follows: two overlapping grains with radii $R_{1}$ and $R_{2}$, mass $m$, normal overlap of $\delta$, normal relative velocity $v$, tangential relative velocity $v_{t}$, and a tangential displacement vector $\delta_{t}$ will experience a normal repulsive force,

$$
F_{n}=\frac{4}{3} Y \sqrt{R^{*}} \delta^{3 / 2}+2 v \sqrt{\frac{5}{6}} \beta \sqrt{S_{n} m},
$$

and a tangential force,

$$
F_{t}=8 G \sqrt{R^{*} \delta} \delta_{t}+2 v_{t} \sqrt{\frac{5}{6}} \beta \sqrt{S_{t} m},
$$

where $R^{*}=R_{1} R_{2} /\left(R_{1}+R_{2}\right), \beta=\frac{\ln (e)}{\ln ^{2}(e)+\pi^{2}}$, and $e$ is the coefficient of restitution. The prefactors $S_{n}=2 Y \sqrt{R^{*} \delta}$ and $S_{t}=8 G \sqrt{R^{*} \delta}$ depend on the Young's modulus $Y$ and the shear modulus $G=\frac{Y}{4(1-v) *(2+v)}$, where $v$ is the Poisson's ratio. The tangential force is truncated according to the value of friction $\mu$ to satisfy the Coulomb condition $F_{t} \leqslant \mu F_{n}$. Beads used in our experiments are made of PMMA with the following material properties: Young's modulus $Y=1.8 \mathrm{GPa}$, Poisson's ratio $v=0.35$, coefficient of restitution $e=0.7$, and density $\rho=1200 \mathrm{~kg} / \mathrm{m}^{3}$. We experimentally measure the coefficient of friction $\mu=0.7 \pm 0.08$. On a technical note, we fix the position of the grains up to four grain diameter from the container walls to ensure that boundary conditions are identical to the experimental ones.

\section{GEOMETRICAL CHARACTERIZATION}

To identify and characterize locally ordered motifs within the packings, we use different local measures based on the spatial position or orientation of grains surrounding any given grain. Such measures are commonly known as the bond order parameter method [38], where the order parameter, $q_{l}$, is defined for each sphere with respect to its fixed number of neighbors. In our calculations, we use $N_{n n}=12$ as the nearest number of neighbors and define $q_{l}$ as follows:

$$
q_{l}(i)=\left(\frac{4 \pi}{2 l+1} \sum_{m=-l}^{m=l}\left(\left|q_{l m}(i)\right|^{2}\right)\right)^{1 / 2},
$$

where $q_{l m}(i)=\frac{1}{N_{n n}(i)} \sum_{j=l}^{N_{n n}(i)}\left(Y_{l m}\left(\mathbf{r}_{\mathbf{i j}}\right)\right)$, with $Y_{l m}$ being the spherical harmonics and $\mathbf{r}_{\mathbf{i j}}$ the vector connecting the central sphere at $\mathbf{r}_{\mathbf{i}}$ to its neighbor at $\mathbf{r}_{\mathbf{j}}$.

Figure 2(a) shows the probability distribution functions (PDF) of the order parameter $q_{6}$ before and after $\phi \approx 0.64$. At a density of $\phi \approx 0.628$, the PDF is wide and has a bell shape; however, in a dense partially crystallized subset $(\phi \approx 0.683)$, two sharp peaks dominate the PDF. These peaks are located at $q_{6}=0.48$ and $q_{6}=0.57$ and correspond to $q_{6}$ values for hcp and fcc crystalline structures, respectively [29,39]. Figure 2(b) shows the dependence of the mean $\left\langle q_{6}\right\rangle$ on the packing density $\phi$. Three regimes are evident from Fig. 2(b) as follows. (i) Below $\phi \approx 0.64,\left\langle q_{6}\right\rangle$ grows slowly. (ii) For packing densities in the range $\phi \in[0.64,0.70],\left\langle q_{6}\right\rangle$ increases sharply, signaling the onset of crystallization around $\phi \approx 0.64$. (iii) As the packing density increases even further $(\phi>0.70),\left\langle q_{6}\right\rangle$ shows some sign of saturation around $\left\langle q_{6}\right\rangle \approx 0.54$ and a high level of fluctuations. These fluctuations are related to the competition between hcp and fcc patterns in highly crystalline packings [39].

We also investigate whether a grain belongs to an hcp-like or fcc-like lattice structure by checking if its $q_{6}$ value falls within the intervals $q_{6}(\mathrm{hcp})=0.48 \pm 0.01$ or $q_{6}(\mathrm{fcc})=0.57 \pm 0.01$. It allows us to measure the ratio of the number of beads belonging to a given crystal motif over the total number of beads in the subsets. The behavior of these ratios versus $\phi$ is shown in Fig. 2(c). Below $\phi_{\text {Bernal }}$, hcp dominates over fcc by almost two orders of magnitude in quantity. While the ratio of hcp motifs grows steadily over the whole density range, the fcc order has a dramatic rise after $\phi_{\text {Bernal }}$ and eventually it dominates over the hcp order in highly crystalline packings $\phi>0.70$. The prevalence of the fcc motifs over hcp patterns remains an outstanding question in dissipative packings of beads [11]. We plan to fully investigate this issue in a followup study.

To further explore the structural changes during crystallization, we now employ a recent extension of the bond order parameter method [40]. It is based on the parameter $q_{l}$ and the Wigner $3 j$ symbols which read

$$
w_{l}(i)=\sum_{\substack{m_{1}, m_{2}, m_{3} \\
m_{1}+m_{2}+m_{3}=0}}\left[\begin{array}{ccc}
l & l & l \\
m_{1} & m_{2} & m_{3}
\end{array}\right] q_{l m_{1}}(i) q_{l m_{2}}(i) q_{l m_{3}}(i) .
$$

The method goes as follow: first, the third-order rotational invariant of rank six, $w_{6}$ is computed, and then we determine the value corresponding to the half-height of its cumulative 

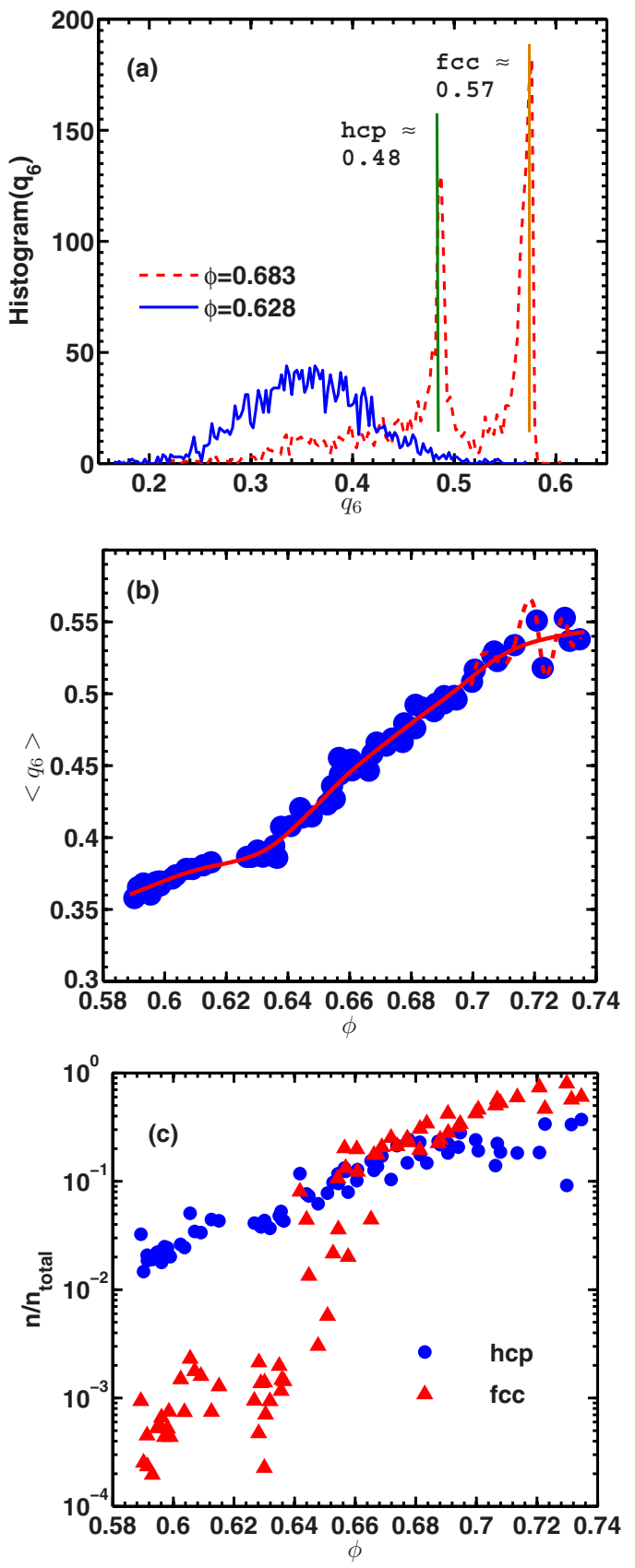

FIG. 2. (Color online) (a) PDF of $q_{6}$ for two distinct packing densities $\phi$. The value of $q_{6}$ in perfect hcp or fcc crystals is $q_{6}(\mathrm{hcp}) \simeq 0.48$ and $q_{6}(\mathrm{fcc}) \simeq 0.57$, respectively. (b) Average of the local orientational parameter $\left\langle q_{6}\right\rangle$ vs packing density $\phi$. (c) The percentage of grains that belong to either hcp or fcc motif vs packing density $\phi$.

distribution function (CDF) referred to as $w_{6}^{h h}$. The CDF of $w_{6}$ is shown in Fig. 3(a) for several subsets over the whole density range. The point where the CDF function reaches its half height, i.e., $w_{6}^{h h}$, differs remarkably for the subsets with $\phi<\phi_{\text {Bernal }}$ and $\phi>\phi_{c}$. In Fig. 3(b) we show the values of $w_{6}^{h h}$ as a function of the packing density. Two clear transitions show up: a sharp rise at $\phi_{\text {Bernal }} \approx 0.64$ and a complete saturation to $w_{6} \approx-0.0125$ beyond $\phi_{c} \approx 0.68$. These transitions have been seen only in numerical simulations [40]. Here, we
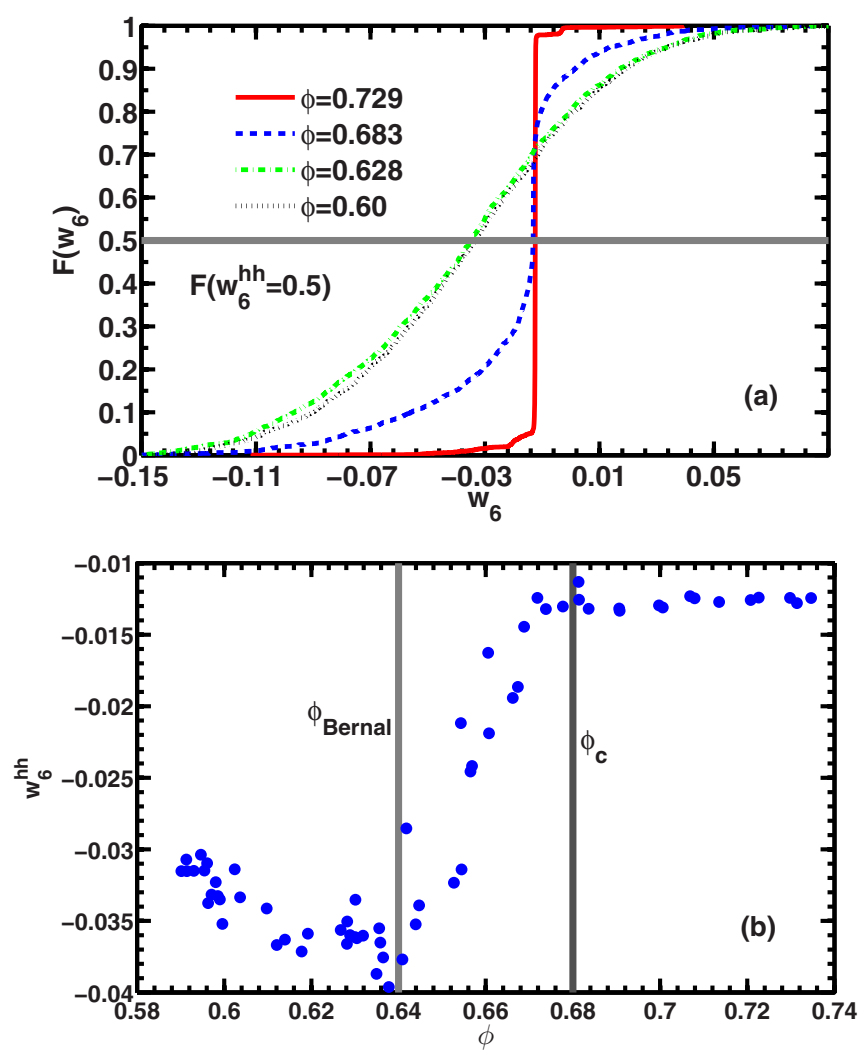

FIG. 3. (Color online) (a) Cumulative distribution function $F\left(w_{6}\right)$ of $w_{6}$ for various packing densities. $w_{6}^{h h}$ is defined as $F\left(w_{6}\right)=$ 0.5. (b) $w_{6}^{h h}$ vs packing fraction $\phi$.

confirm experimentally that $w_{6}^{h h}$ is an extremely sensitive measure of the crystallization onset [40]. Interestingly, we also note the similarities with the transitions observed in the Voronoi volume fluctuations and the topology of packing polytetrahedral structures as observed in [22].

Furthermore, we study the crystallization process through the properties of different structural correlation functions. We first use the cumulative coordination number $Z(r)$, which is defined as

$$
Z(r)=\int_{d}^{d+r} 4 \pi r^{2} g(r) d r,
$$

where $g(r)$ is the radial distribution function (RDF), i.e., the probability distribution of finding the center of a particle at distance $r$ from a reference sphere. $Z(r)$ measures the average number of grains at a radial distance $r$ from a given central grain. In jammed packings ( $\left.\phi<\phi_{\text {Bernal }}\right), Z(r)$ can be accurately described by a power law in the near-contact range $r \in[d, 1.3 d][41]:$

$$
Z(r)=Z_{m}+\gamma(r-d)^{\alpha},
$$

where $Z_{m}$ is the coordination number which characterizes the contacts that bear mechanical forces and $\gamma$ is a constant. We note that this functional form is actually a signature of the power-law singularity in the RDF in the near contact regime [14]. The behavior of $Z(r)$ and $g(r)$ in amorphous packings has been intensely studied during the past two decades [5,14,41]. Here, we measure the evolution of $\alpha$ as the amorphous-crystalline transition takes place. 

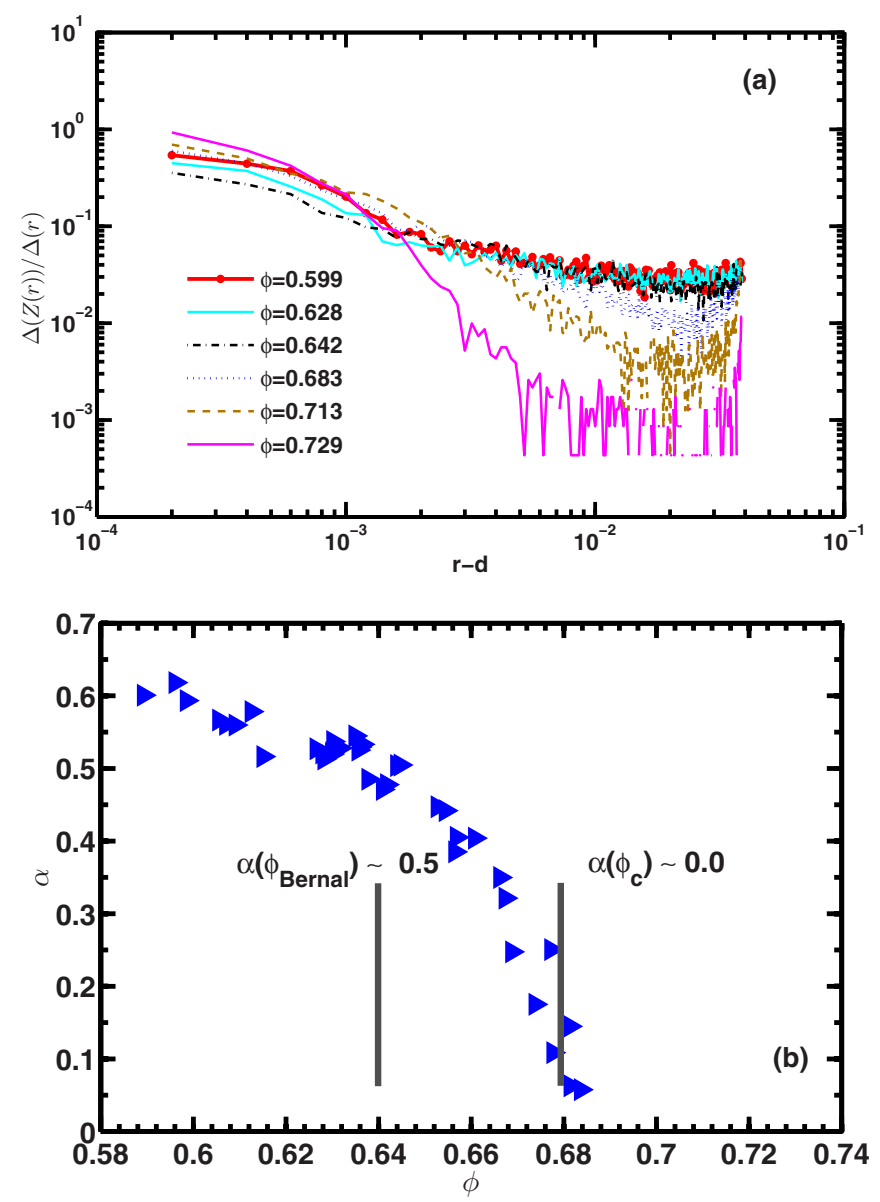

FIG. 4. (Color online) (a) Functional behavior of $\frac{\partial z}{\partial r}$ for six subsets with different packing densities. (b) $\alpha$ vs $\phi$. $\alpha$ can be measured for $\phi<0.68$ from power-law fit of the curves shown in (a).

In Fig. 4(a), we show the behavior of $\frac{\partial z}{\partial r}$ in the near-contact region $r \in[d, 1.3 d]$ for increasing packing densities. Below $\phi_{c} \approx 0.68$, the derivative $\frac{\partial z}{\partial r}$ can be accurately fitted by a power law. Above $\phi_{c}$, the curve develops a pronounced bell shape and the scaling law of Eq. (6) does not hold any more. Figure 4(b) concerns the power-law regime, which shows the exponent $\alpha$ [deduced from the power-law fit in Fig. 4(a)] as a function of $\phi . \alpha$ is slightly larger than 0.5 for $\phi<\phi_{\text {Bernal }}$ and it decreases slowly to 0.5 at $\phi_{\text {Bernal }}$. Beyond $\phi_{\text {Bernal }}, \alpha$ decreases sharply and reaches 0 at $\phi_{c}$. The analysis of $Z(r)$ confirms the presence of two successive structural transitions at $\phi_{\text {Bernal }}$ and $\phi_{c}$.

We now employ the recently developed approach in [28,29] to provide further insights into the grain's neighborhood rearrangements during the crystallization. This analysis assumes that the densely populated neighborhood of each grain, composed of almost-touching neighbors, can be described in terms of a unique geometrical coordination number $Z_{g}$. This approach is based on a modified version of the RDF $g(r)$. The modified radial distribution function (MRDF), $g_{z}(r)$, is made of two Heaviside step functions as formulated below:

$$
g_{z}(r)=\frac{R^{2}}{N r^{2}} \sum_{i=1}^{N} \sum_{j<i}^{N} \Theta\left(\frac{r_{i j}}{r-R}-1\right) \Theta\left(\frac{r+R}{r_{i j}}-1\right)
$$

where $R$ is the radius of spheres, $N$ is the number of beads in the subset, $r_{i j}$ is the distance from the center of particle $i$ to its $j$ th neighbor, and the two Heaviside step functions $\Theta$ check if the criterion $r-R \leqslant r_{i j} \leqslant r+R$ is fulfilled. For instance, if $r$ is increased incrementally, $r=R+n \epsilon$ with $0<\epsilon \ll R$ and $n$ an integer number, then $g_{z}(r)$ counts the number of neighbors in the range $n \epsilon<=r_{i j}<=2 R+n \epsilon$. It corresponds to both the neighbors in true mechanical contact and the number of grains so close that they can be considered as almost-touching neighbors. So $g_{z}(r)$ gives the average number of grains in contact with a virtual particle of radius $r$.

Figure 5(c) shows $g_{z}$ as a function of $\Delta(r)=(r-R) / 2 R$, where $R$ is the mean value of the bead radius in our weakly polydisperse packings. $g_{z}(r)$ first increases with a slight increase of $r$. This is a signature of the fact that each grain neighborhood is densely populated. This primary feature of dense sphere packings contributes to its mechanical stability $[18,42] . g_{z}(r)$ eventually decreases with a further increase of $r$; consequently $g_{z}$ shows a clear maximum as seen in Fig. 5(c). The value of $g_{z}$ at this peak accounts for the grains in mechanical contact as well as the grain neighborhood geometry (i.e., the geometrical neighbors). For this reason, it was used in $[28,29]$ to offer a new perspective on packing geometry and topology by defining a geometrical coordination number $Z_{g}$. We calculate $g_{z}(r)$ and subsequently obtain $Z_{g}$ for each subset by finding its maximum value $\left[Z_{g}=\max \left(g_{z}(r)\right)\right]$. Figure 5(a) shows $Z_{g}$ versus $\phi$ for all subsets. $Z_{g}$ continuously increases over the entire range of packing densities and it can be fitted by $Z_{g}=\alpha 2 \sqrt{3} \phi /(1-\phi)$. This equation was theoretically derived to describe isotropic packings [28]. It, however, seems to remain valid (given a correction factor) even in the case of highly anisotropic crystallized structures.

Figure 5(b) shows $\sigma_{g}$, the standard deviation of the fluctuations in $Z_{g}$, as a function of $\phi$. This graph shows three distinct regimes: (i) below $\phi_{\text {Bernal }}$, where there is a small but almost constant level of fluctuations around $\sigma_{g} \simeq 1.0$; (ii) above $\phi_{\text {Bernal up to }} \phi_{c} \simeq 0.68$ there is a sudden increase in $\sigma_{g}$; this is the density range where both disordered and ordered states coexist in the system; (iii) beyond $\phi>\phi_{c}, \sigma_{g}$ drops sharply as the ordered phase begins to dominate the packing structure.

It has recently been shown $[22,26,27]$ that intense geometrical rearrangements take place in the range $\phi_{\text {Bernal }}<\phi<\phi_{c}$. More precisely, geometrically frustrated polytetrahedral aggregates disassemble as a result of the formation of crystalline nuclei.

The geometrical features of the hard-sphere packings are intimately related to their mechanical properties to ensure the packing stability. The subtle relation that constrains the geometry and the mechanical backbone is one of the most debated questions in the physics of packings $[18,42,43]$. To contribute to this topic, we now investigate the effects of crystallization on the mechanical features both at the local grain scale and globally.

\section{MECHANICAL PROPERTIES}

The mechanical contacts through which intergranular forces are exchanged are an essential predictor of the mechanical properties of a granular assembly. The highly accurate 

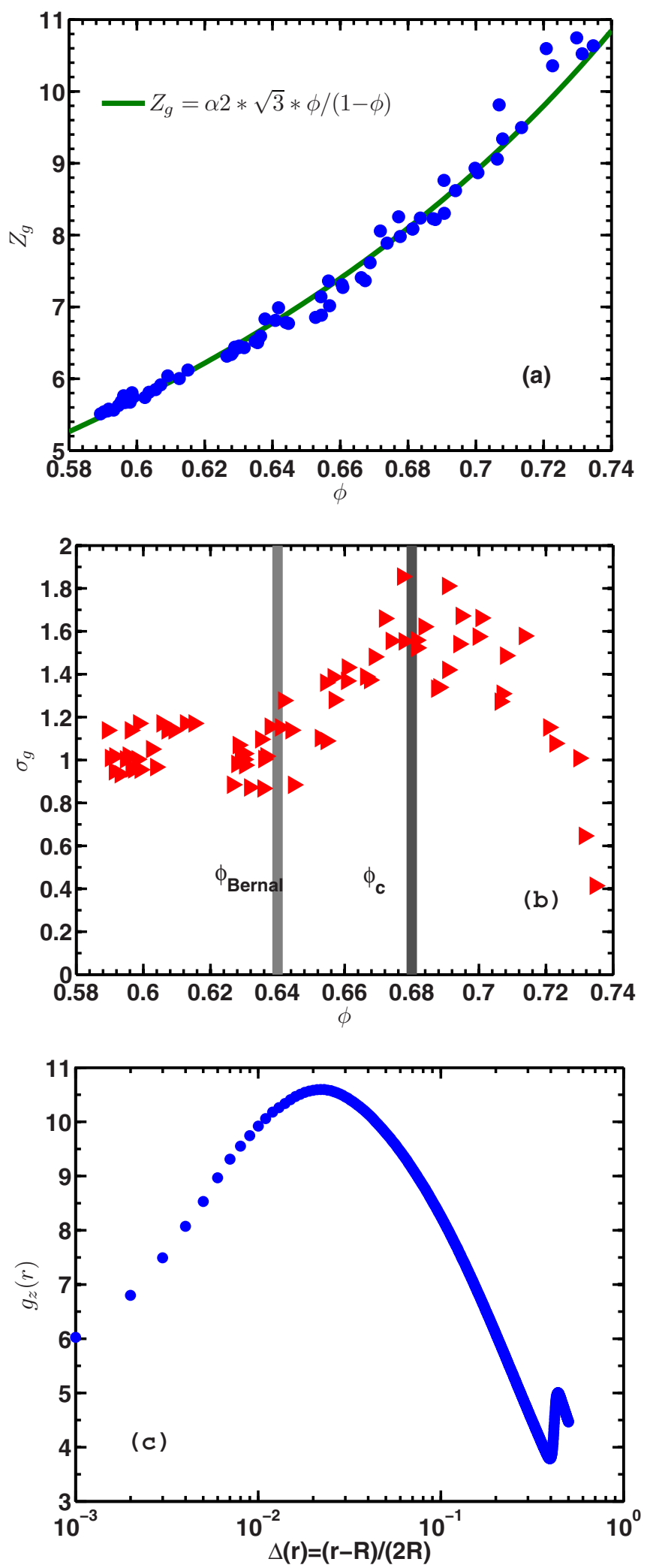

FIG. 5. (Color online) (a) Geometrical coordination number $Z_{g}$ vs packing density $\phi . Z_{g}$ is averaged over 4000 bead subsets. The geometrical coordination number is fitted by $Z_{g}=\alpha 2 \sqrt{3} \phi /(1-\phi)$ with a prefactor $\alpha \simeq 1.1$ added to the formula derived in [28]. (b) Standard deviation $\sigma_{g}$ vs $\phi$. (c) Typical behavior of the modified radial distribution function $g_{z}(\Delta(r))$ as a function of $\Delta(r)=(r-R) / 2 R$.
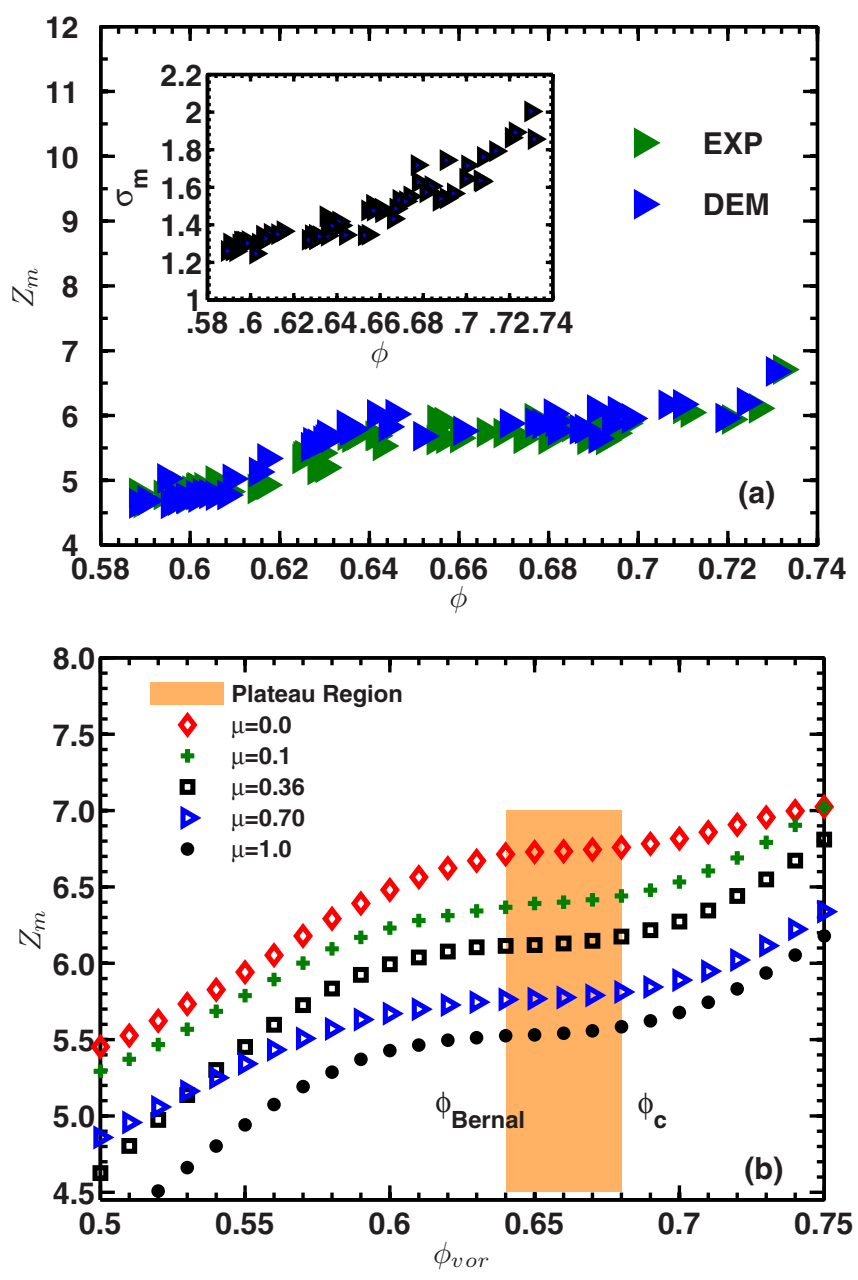

FIG. 6. (Color online) (a) Mechanical coordination number $Z_{m}$ vs packing density $\phi$ measured both from experimental data by evaluating grain overlaps and from the DEM data after numerical relaxation. In the DEM code, the friction coefficient is set to its experimental value $\mu=0.70$. Inset: standard deviation $\sigma_{m}$ of $Z_{m}$ vs $\phi$. (b) $Z_{m}$ averaged at the grain scale vs the local density $\phi_{V o r}=\frac{1 / 6 \pi d^{3}}{V_{v o r}}$, where $V_{v o r}$ is the volume of each Voronoi cell.

experimental data collected on the grain positions and radii allows us to calculate the force bearing (mechanical) contacts directly from raw experimental data by determining the grain overlaps. Additionally, using the DEM simulations as described previously, we can calculate the full intergranular forces with numerical precision. Moreover, it allows us to virtually explore the influence of the friction coefficient $\mu$ by tuning it directly in the DEM code.

\section{A. Mechanical coordination number $Z_{m}$}

First we investigate the behavior of the mechanical coordination number $Z_{m}$ which characterizes the contacts that bear forces. This descriptor is directly estimated from the measurement of grain overlap. Figure 6(a) shows the evolution of $Z_{m}$ with the subset packing density $\phi$. It also compares the experimental and the DEM results. Clearly $Z_{m}$ evolves differently than $Z_{g}$ [see Fig. 5(a)]. In disordered packings, $\phi<\phi_{\text {Bernal }}, Z_{m}$ grows with the packing density. The onset 
of crystallization coincides with a clear change of trend in $Z_{m} . Z_{m}$ shows a plateau for $0.64 \leqslant \phi \leqslant 0.68$. We measure $Z_{\text {mplat }} \approx 5.6$. Beyond $\phi_{c} \simeq 0.68, Z_{m}$ increases again up to $Z_{m} \simeq 6.8$ at $\phi \simeq 0.73$. In these highly crystalline packings, the difference between $Z_{g}$ and $Z_{m}$ is the largest. We emphasize that this behavior of $Z_{m}$ is supported by direct experimental measurements based on the estimation of grain overlaps as well as the DEM results obtained with numerical precision. It should be noted that the plateau behavior of $Z_{m}$ has only been reported in numerical simulations of frictionless packings [29]. Our results recover this important mechanical feature in experimental frictional packings, thus proving some level of universality of this feature.

In Fig. 6(a), $Z_{m}$ and the packing fraction are averaged over 4000 grains. However, $Z_{m}$ can also be measured at the scale of individual grains. Figure 6(b) shows the evolution of $Z_{m}$ versus the local packing density, $\phi_{V o r}$, defined as $\phi_{V o r}=\frac{1 / 6 \pi d^{3}}{V_{v o r}} . V_{v o r}$ is the volume surrounding a grain measured via the Voronoi tessellation of the packing $[22,44]$. The trend of $Z_{m}$ versus $\phi_{V o r}$ is similar to that observed in Fig. 6(a). There is a clear plateau in the density range $\left[\phi_{\text {Bernal }}, \phi_{c}\right]$.

The DEM code allows us to tune the friction coefficient. On a technical note, we use the same initial packing configuration, namely the one measured experimentally, and the value of $\mu$ is modified in the code before starting the numerical relaxation. Thus we have the ability to test the robustness of the mechanical plateau with respect to packing dissipative properties. Figure 6(b) demonstrates that the plateau in $Z_{m}$ is a resilient mechanical feature of the packing structure. However, the value of the plateau is friction dependent: $Z_{\text {mplat }} \approx 6.7$ for $\mu=0 ; Z_{\text {mplat }} \approx 5.5$ for $\mu=1$.

An equally important mechanical quantity is the standard deviation $\sigma_{m}$ of $Z_{m}$, which is an indication of the fluctuations in the force network and the mechanical backbone. The inset of Fig. 6(a) shows that $\sigma_{m}$ increases steadily with $\phi$ and eventually peaks at $\sigma_{m} \simeq 2.0$ for $\phi \simeq 0.73$. This high level of fluctuations for $\sigma_{m}$ for the crystalline state $\left(\phi>\phi_{c}\right)$, along with a significant drop in the geometric fluctuations $\left(\sigma_{g}\right)$ in this density range, suggests that a geometrically ordered structure still possesses a highly random mechanical backbone.

\section{B. Topology of force network}

The mechanical coordination number $\mathrm{Zm}$ is an essential component of the mechanical properties of a granular assembly. Yet it does not contain information on the global topology of the force network, nor information on the intensity of the force transmitted through the contact. The structure and
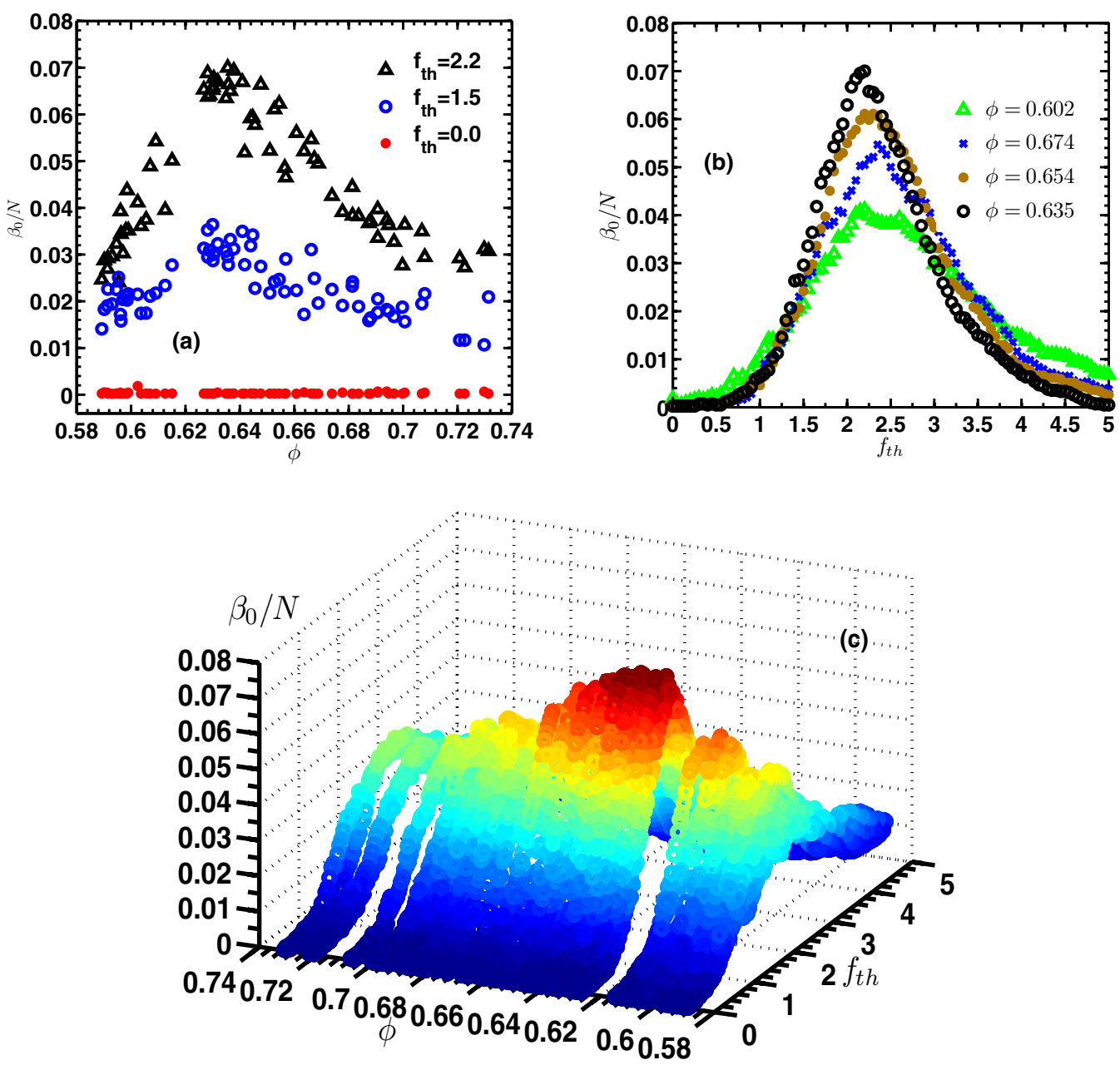

FIG. 7. (Color online) $\beta_{0}$ is normalized by the number of spheres in the subset $N$. (a) $\beta_{0} / N$ vs packing fraction for different force thresholds. (b) $\beta_{0} / N$ vs force threshold $f_{t h}$ for different packing densities. (c) Evolution of $\beta_{0} / N$ in the phase space $\left(\phi, f_{t h}\right)$. 
topology of the network of intergranular forces play a crucial role in the mechanical stability of a granular system, which is an extremely rich nonlinear problem [5,24]. The intricate and complex network of force transmitting grain contacts governs the rich aspects of the jamming transition [23] and gives the granular materials their unique characteristics such as soft modes [5].

Having access to the intergranular forces in our packings, we aim to quantify the connectivity of the global force network and its evolution as the crystallization takes place. We use a topological invariant called the Betti numbers $\beta[45,46]$, whose first component, $\beta_{0}$, calculates the number of clusters formed from mutually connected beads.

In order to relate this topological quantity to the mechanics of our granular systems, we take the following procedure. (I) In each subset, the value of the normal forces between each pair of grains is normalized to the mean value of the normal force in the entire corresponding subset: $f_{n}=\frac{F_{n}}{\left\langle F_{n}\right\rangle}$. (II) A force threshold, $f_{t h}$, is defined and we count the number of isolated clusters formed by mutually connected particles with $f_{n} \geqslant f_{t h}$. (III) The number of isolated clusters is counted for different values of $f_{t h}$ in each subset. For $f_{t h}=0.0, \beta_{0}$ simply measures the network of mechanical contacts. As $f_{t h}$ increases, particles whose mechanical contacts carry forces below $f_{t h}$ are eliminated from this network, changing its topology.

Figure 7(a) shows the zeroth Betti number normalized by the number of spheres in each subset. For the force thresholds $f_{t h} \gtrsim 1.5, \beta_{0}$ is a sensitive parameter of $\phi$, and for $f_{t h} \simeq 2.2$ the trend of $\beta_{0}$ is remarkably peaked around $\phi_{\text {Bernal. This }}$ distinct behavior of $\beta_{0}$ is shown more clearly in Fig. 7(b), where $f_{t h}$ is varied over a large range in four subsets. This figure shows that there is a characteristic $f_{\text {th }}$ range $(\approx 2.0<$ $f_{t h}<\approx<2.5$ ), in which the number of isolated componets of the force networks is the largest. $\beta_{0}$ decreases with further increase of the force threshold as less contacts fulfill the criterion of $f_{n}>f_{t h}$. Figure 7(c) encapsulates the variation of both $\phi$ and $f_{t h}$ across all subsets. This phase diagram reveals that for the characteristic $f_{t h}$ range, large and small forces are distributed more uniformly (homogeneously) near $\phi_{\text {Bernal }}$. In other words, large and small forces are homogeneously mixed inside the force network and as a result, thresholding the force produces a large number of connected components. For $\phi$ above $\phi_{c} \simeq 0.68$, the declining trend of $\beta_{0}$ is mostly similar to that observed in the range $0.58<\phi<0.61$ implying that forces are distributed more unhomogeneously. This further confirms the observation [see Fig. 6(a)] that even in regions of highly crystallized order the topology of force network and the mechanical backbone may resemble that of disordered states.

\section{CONCLUSION}

In this paper, we have conducted an exhaustive investigation of the crystallization process in experimentally produced hard-sphere packings. We combined experiments with stateof-the-art 3D imaging and numerical simulation techniques to understand the transition from disordered to ordered state in terms of geometrical, mechanical and topological changes.

We have shown that for packing densities $0.64<\phi<0.68$ the local orientational order emerges in the system and gradually dominates the entire packing when $\phi>0.68$. The region $0.64<\phi<0.68$ is geometrically characterized by a steep increase in the fluctuations of the geometrical coordination number, $Z_{g}$. These fluctuations are greatly reduced for $\phi>0.68$ as highly ordered structures become dominant in the system. These results are in agreement with previous studies where large topological changes have been observed for $0.64<\phi<0.68$ as a result of the disassembling of the polytetrahedral structures [22].

In terms of the system's mechanics, we have shown that the density range of $0.64<\phi<0.68$ is accompanied by drastic changes in the behavior of the mechanical coordination number, $Z_{m}$. Crucially, for $0.64<\phi<0.68$, where both disordered and ordered states coexist, $Z_{m}$ reaches a plateau. The phenomenon is robust and has been observed over a broad range of values for the friction coefficient. This feature of crystallization in frictional packings is reminiscent of some classic aspects of first-order phase transition in equilibrium thermodynamics [29].

Finally, we have shown that in almost fully ordered structures, $Z_{m}$ and the topology of the force network show a high level of fluctuation. This behavior indicates the persistence of a random mechanical backbone in highly crystallized packings. These observations support the conclusions drawn in recent numerical studies where the mechanical properties of lattices made of weakly polydisperse beads [21] or jammed lattice sphere packings in high dimensions [47] have been shown to share similarities with those of amorphous packings. These findings might also help in better understanding the rheology of complex granular or suspension systems $[48,49]$. In conclusion, these results give hope for the elaboration of basic principles for granular crystallization.

\section{ACKNOWLEDGMENTS}

We thank T. J. Senden for his support. N.F. thanks M. Driol for her support. The authors thank E. Franklin for comments on the manuscript. The works of M.F. and S.M.V.A. were supported in part by the Research Council of the University of Tehran.
[1] J.-F. Sadoc and R. Mosseri, Geometrical Frustration (Cambridge University Press, Cambridge, UK, 1999).

[2] R. G. Larson, The Structure and Rheology of Complex Fluids (Oxford University Press, Oxford, 1998).

[3] G. Parisi and F. Zamponi, Rev. Mod. Phys. 82, 789 (2010).

[4] J. D. Bernal, Proc. R. Soc. A 280, 299 (1964).
[5] M. van Hecke, J. Phys.: Condens. Matter 22, 033101 (2010).

[6] O. Pouliquen, M. Nicolas, and P. D. Weidman, Phys. Rev. Lett. 79, 3640 (1997).

[7] J. S. Olafsen and J. S. Urbach, Phys. Rev. Lett. 95, 098002 (2005). 
[8] F. Pacheco-Vázquez, G. A. Caballero-Robledo, and J. C. RuizSuárez, Phys. Rev. Lett. 102, 170601 (2009).

[9] P. M. Reis, R. A. Ingale, and M. D. Shattuck, Phys. Rev. Lett. 96, 258001 (2006).

[10] K. E. Daniels and R. P. Behringer, Phys. Rev. Lett. 94, 168001 (2005).

[11] A. Panaitescu, K. A. Reddy, and A. Kudrolli, Phys. Rev. Lett. 108, 108001 (2012).

[12] A. Prevost, P. Melby, D. A. Egolf, and J. S. Urbach, Phys. Rev. E 70, 050301 (2004).

[13] G. Y. Onoda and E. G. Liniger, Phys. Rev. Lett. 64, 2727 (1990).

[14] T. Aste, M. Saadatfar, and T. J. Senden, Phys. Rev. E 71, 061302 (2005).

[15] M. Jerkins, M. Schröter, H. L. Swinney, T. J. Senden, M. Saadatfar, and T. Aste, Phys. Rev. Lett. 101, 018301 (2008).

[16] S. Torquato, T. M. Truskett, and P. G. Debenedetti, Phys. Rev. Lett. 84, 2064 (2000).

[17] P. Chaudhuri, L. Berthier, and S. Sastry, Phys. Rev. Lett. 104, 165701 (2010).

[18] P. Charbonneau, E. I. Corwin, G. Parisi, and F. Zamponi, Phys. Rev. Lett. 109, 205501 (2012).

[19] S. C. Kapfer, W. Mickel, K. Mecke, and G. E. Schröder-Turk, Phys. Rev. E 85, 030301 (2012).

[20] A. J. Liu and S. R. Nagel, Nature (London) 396, 21 (1998).

[21] R. Mari, F. Krzakala, and J. Kurchan, Phys. Rev. Lett. 103, 025701 (2009).

[22] N. Francois, M. Saadatfar, R. Cruikshank, and A. Sheppard, Phys. Rev. Lett. 111, 148001 (2013).

[23] D. Bi, J. Zhang, B. Chakraborty, and R. P. Behringer, Nature (London) 480, 355 (2011).

[24] T. S. Majmudar and R. P. Behringer, Nature (London) 435, 1079 (2005).

[25] S. Papanikolaou, C. S. O’Hern, and M. D. Shattuck, Phys. Rev. Lett. 110, 198002 (2013).

[26] A. V. Anikeenko and N. N. Medvedev, Phys. Rev. Lett. 98, 235504 (2007).

[27] A. V. Anikeenko, N. N. Medvedev, and T. Aste, Phys. Rev. E 77, 031101 (2008).

[28] C. Song, P. Wang, and H. A. Makse, Nature (London) 453, 629 (2008).
[29] Y. Jin and H. A. Makse, Physica A 389, 5362 (2010).

[30] M. Hanifpour, N. Francois, S. M. Vaez Allaei, T. Senden, and M. Saadatfar, Phys. Rev. Lett. 113, 148001 (2014).

[31] P. Philippe and D. Bideau, Phys. Rev. Lett. 91, 104302 (2003).

[32] T. Aste, M. Saadatfar, and T. J. Senden, J. Stat. Mech. (2006) P07010.

[33] M. Saadatfar, N. Francois, A. Arad et al., SEG Las Vegas Annual Meeting, 2012 (unpublished), pp. 2273-2281.

[34] T. Varslot, A. Kingston, G. Myers, and A. Sheppard, Med. Phys. 38, 5459 (2011).

[35] M. Saadatfar, A. P. Sheppard, T. J. Senden, and A. J. Kabla, J. Mech. Phys. Solids 60, 55 (2012).

[36] G. W. Delaney, T. D. Matteo, and T. Aste, J. Soft Matter 6, 2992 (2010).

[37] http://www.cfdem.com/liggghts

[38] P. J. Steinhardt, D. R. Nelson, and M. Ronchetti, Phys. Rev. Lett. 47, 1297 (1981).

[39] B. A. Klumov, Y. Jin, and H. Makse, J. Phys. Chem. B 118, 10761 (2014).

[40] B. A. Klumov, S. A. Khrapak, and G. E. Morfill, Phys. Rev. B 83, 184105 (2011).

[41] A. Donev, S. Torquato, and F. H. Stillinger, Phys. Rev. E 71, 011105 (2005).

[42] M. Wyart, Phys. Rev. Lett. 109, 125502 (2012).

[43] C. F. Moukarzel, Phys. Rev. Lett. 81, 1634 (1998).

[44] F. M. Schaller, M. Neudecker, M. Saadatfar, G. W. Delaney, G. E. Schröder-Turk, and M. Schröter, Phys. Rev. Lett. 114, 158001 (2015).

[45] V. Robins, in Morphology of Condensed Matter: Physics and Geometry of Spatially Complex Systems, edited by K. Mecke and D. Stoyan, Lecture Notes in Physics Vol. 600 (Springer, Berlin, Heidelberg, 2002), pp. 261-274.

[46] L. Kondic, A. Goullet, C. S. O'Hern, M. Kramar, K. Mischaikow, and R. P. Behringer, Europhys. Lett. 97, 54001 (2012).

[47] Y. Kallus, E. Marcotte, and S. Torquato, Phys. Rev. E 88, 062151 (2013).

[48] F. Boyer, E. Guazzelli, and O. Pouliquen, Phys. Rev. Lett. 107, 188301 (2011).

[49] Y. Forterre and O. Pouliquen, Annu. Rev. Fluid Mech. 40, 1 (2008). 